\title{
Efficacy and Safety of the New Appetite Suppressant, Liraglutide: A Meta-Analysis of Randomized Controlled Trials
}

\author{
Shinje Moon ${ }^{1}$, Jibeom Lee ${ }^{2}$, Hye Soo Chung ${ }^{1}$, Yoon Jung Kim ${ }^{1}$ Jae Myung Yu ${ }^{1}$, Sung Hoon Yu ${ }^{3}$, Chang-Myung $\mathrm{Oh}^{2}$ \\ ${ }^{1}$ Department of Endocrinology and Metabolism, Hallym University College of Medicine, Chuncheon; ${ }^{2}$ Department of \\ Biomedical Science and Engineering, Gwangju Institute of Science and Technology, Gwangju; ${ }^{3}$ Division of Endocrinology and \\ Metabolism, Department of Internal Medicine, Hanyang University Guri Hospital, Hanyang University College of Medicine, \\ Guri, Korea
}

Background: Obesity is a chronic disease associated with metabolic diseases such as diabetes and cardiovascular disease. Since the U.S. Food and Drug Administration approved liraglutide as an anti-obesity drug for nondiabetic patients in 2014, it has been widely used for weight control in overweight and obese people. This study aimed to systematically analyze the effects of liraglutide on body weight and other cardiometabolic parameters.

Methods: We investigated articles from PubMed, EMBASE, and the Cochrane Library to search randomized clinical trials that examined body weight changes with liraglutide treatment.

Results: We included 31 studies with 8,060 participants for this meta-analysis. The mean difference (MD) between the liraglutide group and the placebo group was $-4.19 \mathrm{~kg}(95 \%$ confidence interval [CI], -4.84 to -3.55$)$, with a $-4.16 \%$ change from the baseline $(95 \% \mathrm{CI},-4.90$ to -3.43$)$. Liraglutide treatment correlated with a significantly reduced body mass index (MD: $-1.55 ; 95 \% \mathrm{CI},-1.76$ to -1.34 ) and waist circumference (MD: $-3.11 \mathrm{~cm} ; 95 \% \mathrm{CI},-3.59$ to -2.62 ) and significantly decreased blood pressure (systolic blood pressure, MD: $-2.85 \mathrm{~mm} \mathrm{Hg}$; 95\% CI, -3.36 to -2.35 ; diastolic blood pressure, MD: $-0.66 \mathrm{~mm} \mathrm{Hg}$; $95 \% \mathrm{CI},-1.02$ to -0.30 ), glycated hemoglobin (MD: $-0.40 \% ; 95 \% \mathrm{CI},-0.49$ to -0.31 ), and low-density lipoprotein cholesterol (MD: $-2.91 \mathrm{mg} / \mathrm{dL} ; 95 \% \mathrm{CI}$, -5.28 to -0.53 ; MD: $-0.87 \%$ change from baseline; $95 \% \mathrm{CI},-1.17$ to -0.56 ).

Conclusion: Liraglutide is effective for weight control and can be a promising drug for cardiovascular protection in overweight and obese people.

Keywords: Liraglutide; Glucagon-like peptide 1; Obesity; Metabolic syndrome; Meta-analysis

Received: 7 December 2020, Revised: 17 March 2021, Accepted: 16 April 2021

Corresponding authors: Sung Hoon $\mathrm{Yu}$

Division of Endocrinology and Metabolism, Department of Internal Medicine, Hanyang University Guri Hospital, Hanyang University College of Medicine, 153 Gyeongchun-ro, Guri 11923, Korea

Tel: +82-31-560-2154, Fax: +82-31-560-2189, E-mail: physicianyu@daum.net

Department of Biomedical Science and Engineering, Gwangju Institute of Science and Technology, 123 Cheomdangwagi-ro, Buk-gu, Gwangju 61005, Korea Tel:+82-62-715-5377, Fax: +82-62-715-5377, E-mail: cmoh@gist.ac.kr 


\section{INTRODUCTION}

Obesity is a chronic disease associated with metabolic diseases such as diabetes, cardiovascular disease, chronic kidney disease, and cancer [1]. The rising prevalence of obesity has led to its recognition as a serious public health problem worldwide [2]. Lifestyle modification is the first step in controlling body weight in overweight and obese individuals [3]. Although lifestyle modification is the most effective strategy to manage body weight and prevent the metabolic complications of obesity, compliance with a healthy lifestyle proves difficult for many individuals. Dalle Grave et al. [4] reported that, despite the efficacy of lifestyle modification for reducing body weight, $70 \%$ to $80 \%$ of patients treated with lifestyle modification failed to maintain a reduced body weight at 3 to 5 years.

Pharmacotherapy is the next best option for weight control. The U.S. Food and Drug Administration (FDA) has approved four agents (orlistat, naltrexone-bupropion, phentermine-topiramate, and liraglutide) for long-term weight control, and of these, phentermine-topiramate and liraglutide are the most effective agents for weight control [5]. Liraglutide is a long-acting ana$\log$, with $87 \%$ homology to human glucagon-like peptide 1 (GLP-1), that acts as a GLP-1 receptor agonist [6].This drug was originally used only for glycemic control for people with diabetes. However, after several clinical trials reported a weightreduction effect without hypoglycemia in obese people without diabetes [7], the FDA approved liraglutide for the treatment of obesity in 2014.

Many human and animal studies have shown the beneficial effects of GLP-1 receptor agonists in the brain and in peripheral tissues, besides their glucose-lowering effects [7,8]. GLP-1 receptor agonists increase insulin secretion from pancreatic beta cells, reduce insulin resistance and gluconeogenesis in the liver, slow gastric emptying, and reduce appetite [7]. This study aimed to investigate the effects of liraglutide on body weight and cardiovascular benefits. Therefore, we performed a metaanalysis of randomized controlled trials (RCTs) to assess liraglutide treatment in overweight and obese individuals.

\section{METHODS}

\section{Search strategy}

The literature search was performed according to the Preferred Reporting Items for Systematic Reviews and Meta-Analyses guidelines (Supplemental Table S1) [9], and the data were extracted by two researchers (S.M. and C.M.O.). S.M. extracted data from citation databases, including PubMed, EMBASE, and the Cochrane Library, from the inception of the database to March 7, 2021, and C.M.O. crosschecked these data for accuracy. Search terms included combinations of "liraglutide" and "obesity."

\section{Study selection}

The inclusion criteria were as follows: (1) population: participants who were overweight or obese (body mass index [BMI] $\left.\geq 25 \mathrm{~kg} / \mathrm{m}^{2}\right)$; (2) intervention: 1.8 or $3.0 \mathrm{mg}$ liraglutide injection daily for 4 weeks or more; (3) comparators: control group with placebo; (4) outcomes: data on changes in the following variables: weight, waist circumference (WC), BMI, blood pressure, glycated hemoglobin (HbAlc), or low-density lipoprotein cholesterol (LDL-C); and (5) study design: RCTs.

The exclusion criteria were as follows: (1) articles on animal studies or in vivo experiments, only abstracts, and non-original articles, including expert opinions or reviews; (2) non-RCT studies; (3) studies on non-obese patients; and (4) studies involving participants with diseases that could affect weight change.

\section{Data extraction}

The following variables were extracted from the articles selected by two researchers (S.M. and C.M.O.) using the same criteria: first author, publication year, characteristics of the participants, number of study participants, mean age, weight, WC, BMI, blood pressure, HbA1c, and LDL-C.

\section{Quality assessment}

Two researchers (S.M. and C.M.O.) evaluated the quality of RCTs using the "Revised Cochrane risk-of-bias tool for randomized trials (ROB-2.0)." Discrepancies were resolved through discussions with a third investigator (S.H.Y.).

\section{Data analyses and statistical methods}

The pooled effect sizes were presented as mean differences (MD) and 95\% confidence intervals (CIs) between the intervention group and the placebo group using the random effects model. Because the weight and LDL-C levels were reported by two different parameters, such as mean change or percentage change from baseline, we calculated the standard mean differences (SMD) of these variables. The heterogeneity between studies was tested using Cochrane Q statistic and Higgins $I^{2}$ statistic; an $I^{2}$ greater than $50 \%$ was considered indicative of heterogeneity between studies. Publication bias was evaluated using the funnel 
plot and Egger's test, and sensitivity analyses were conducted between studies. In addition, subgroup analysis was performed based on the dosage of liraglutide and the presence of diabetes. All analyses were conducted using Comprehensive Meta-Analysis software version 3 (Biostat, Englewood, NJ, USA).

\section{RESULTS}

\section{Study characteristics}

A total of 2,591 articles (PubMed 844, EMBASE 1,153, Cochrane Library 594) were identified on the literature search; 676 overlapping articles were excluded, and 1,915 articles were verified for further screening. After excluding articles that did not meet the inclusion criteria, 101 studies were assessed for eligibility. After further review and quality assessment, 31 studies were included in the meta-analysis (Fig. 1) [10-39], and a total of 8,060 participants were included. There were three and 28 studies in obese adolescents and adults, respectively. Nine of the studies in adults only included obese or overweight patients with diabetes, and 12 studies included obese or overweight patients without diabetes. The main baseline characteristics of each study are summarized in Table 1 [10-40]. In the quality assessment, the risk-of-bias was low in 27 studies [10-15,17-22,
24-28,30-37,39]. Four RCTs had some concerns with regard to bias arising because of deviations from the intended interventions $[16,23,29,38]$.

\section{Effect of liraglutide on changes in anthropometric data}

Twenty-four studies [10-14,16-22,24,25,27,28,30-34,37-39] with 7,742 participants (liraglutide group 4,721; placebo group $3,021)$ reported changes in weight from the baseline. The SMD between the liraglutide group and the placebo group on using a random effects model was $-0.71(95 \% \mathrm{CI},-0.81$ to -0.61$)$, which indicated significantly more weight loss in the liraglutide group, and the $I^{2}$ was $65.6 \%$, indicating significant heterogeneity (Fig. 2). The funnel plot was symmetrical, and publication bias was not detected (Egger's test: $P=0.97$ ) (Supplemental Fig. S1). In sensitivity analysis, the significance of the results did not change even after each study was removed, and no outliers were observed (Supplemental Fig. S2).

Twenty one studies [12-14,16-22,24,25,28,30-34,37-39] with 6,228 participants (liraglutide group 3,756; placebo group $2,472)$ reported changes in weight $(\mathrm{kg})$ from the baseline (MD, $-4.19 \mathrm{~kg} ; 95 \% \mathrm{CI},-4.84$ to -3.55$)$. Thirteen studies [10$13,16,18,19,25,27,28,32-34]$ with 6,699 participants (liraglutide group 4,146; placebo group 2,553) reported percentage changes

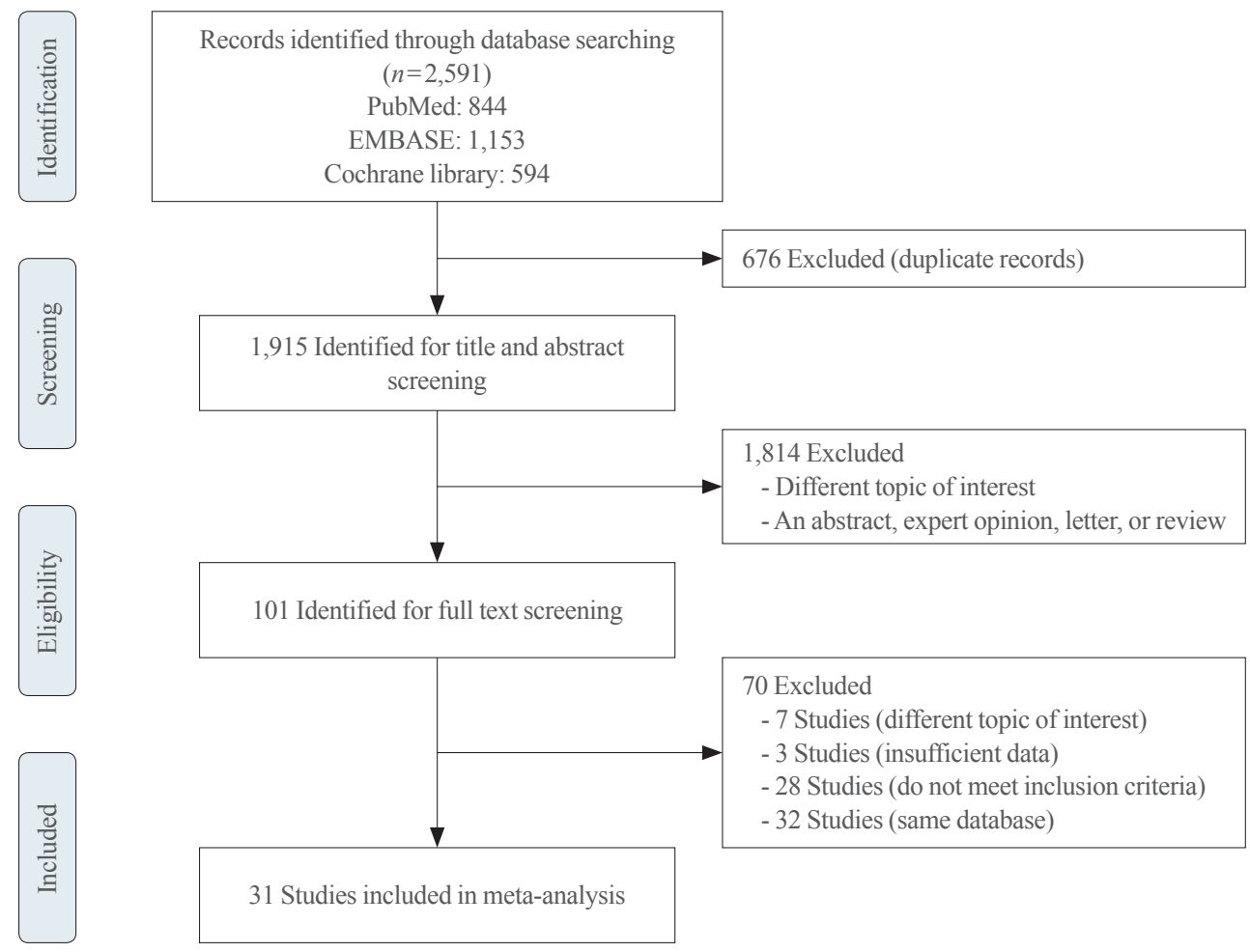

Fig. 1. Schema of the search strategy. 


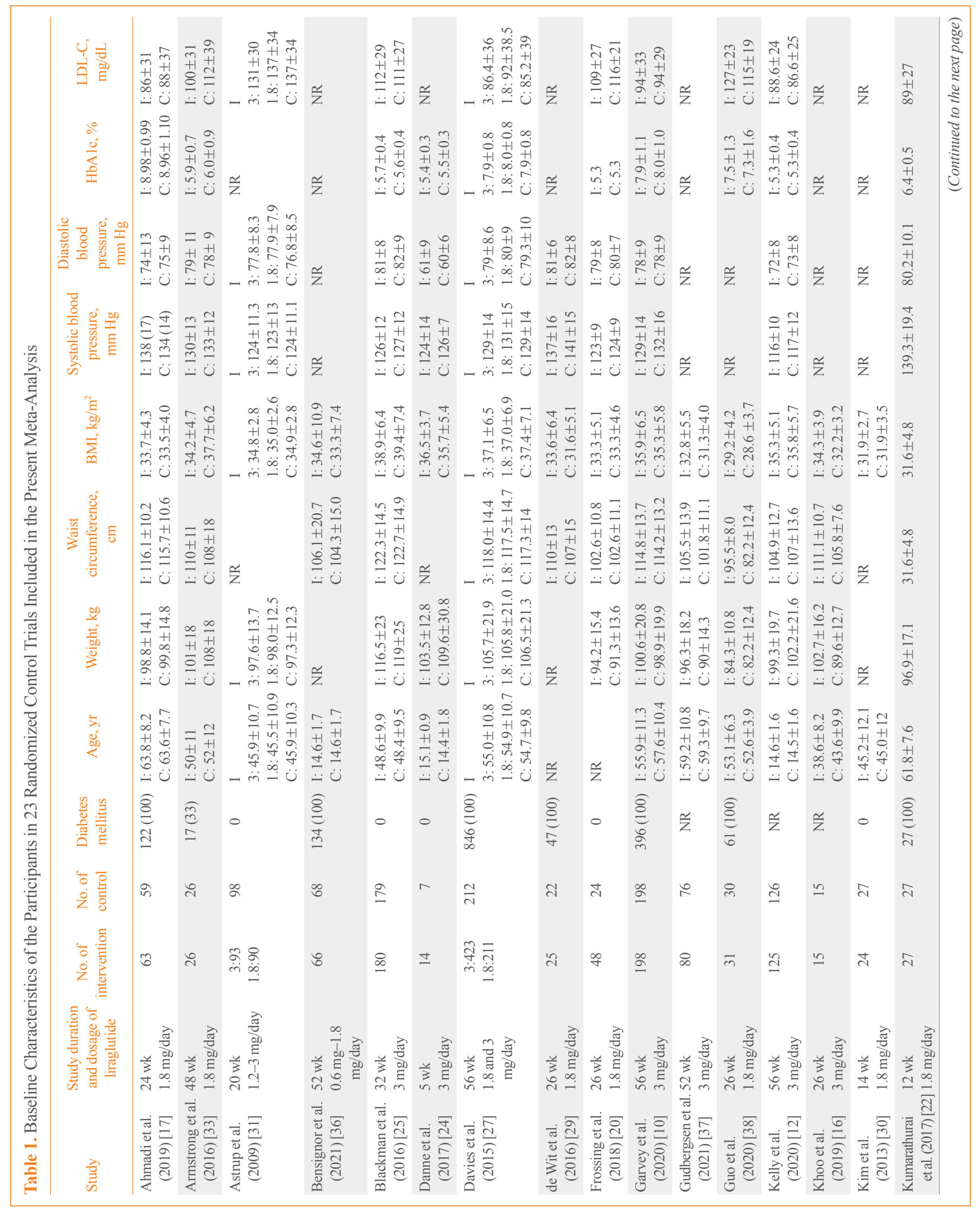




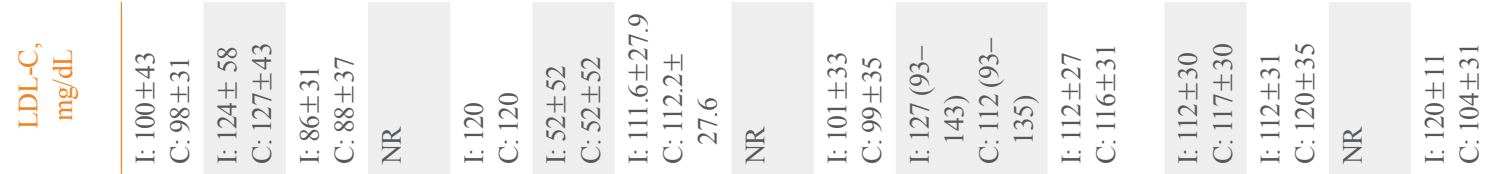

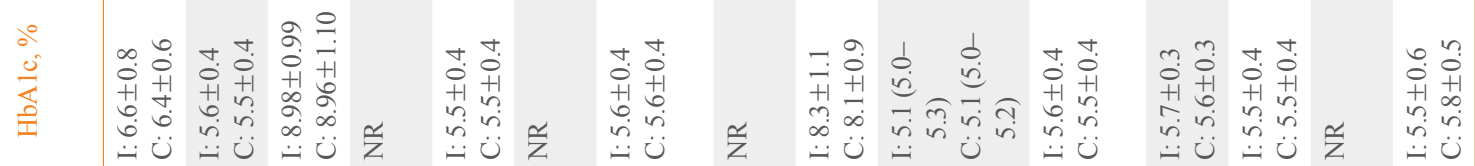

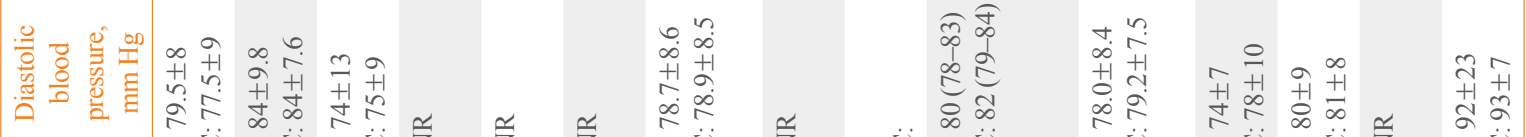

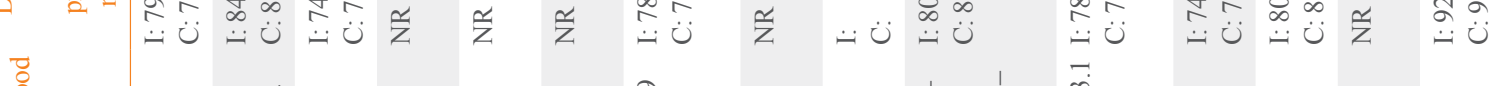

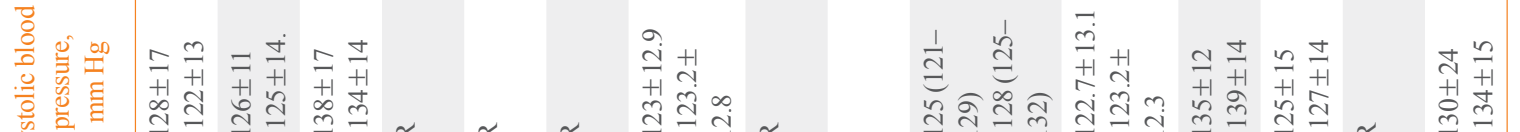

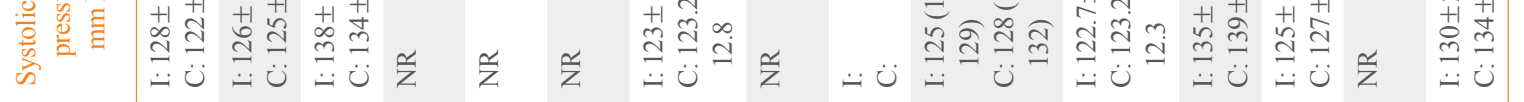

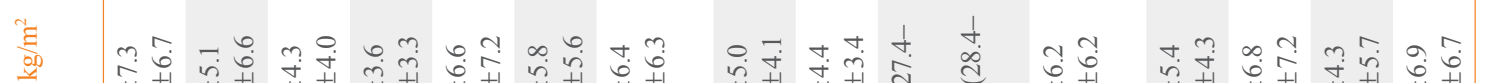

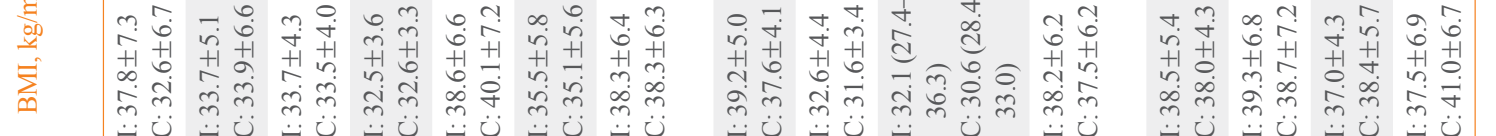

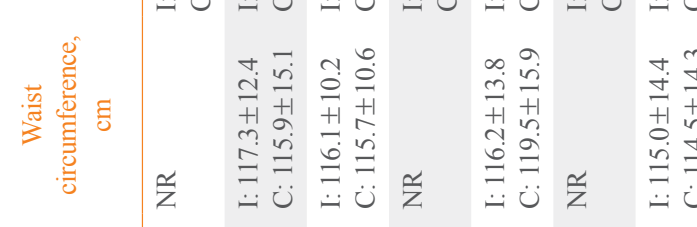

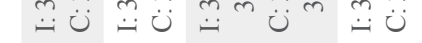

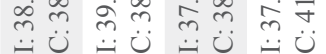
으 $n$ ก

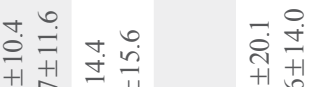

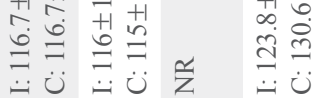

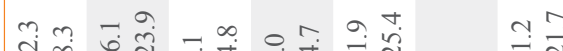

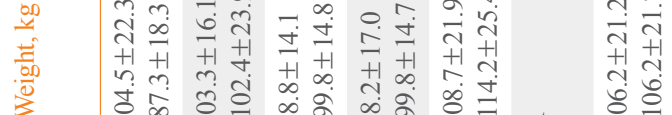

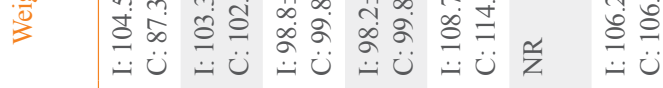

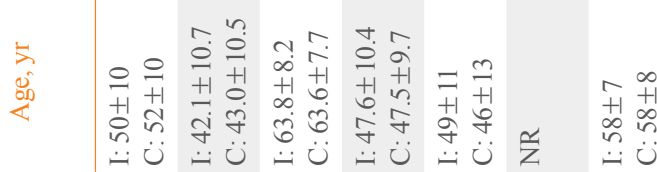

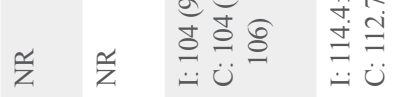

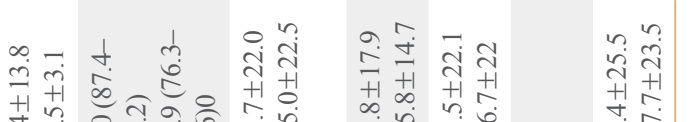

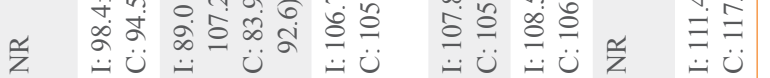
荡 $\frac{0}{0}$

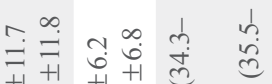

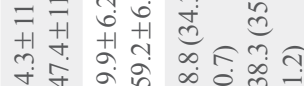

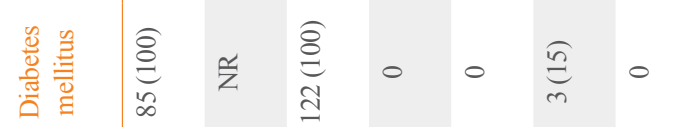

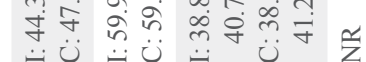

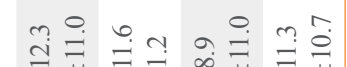
H

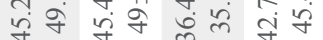

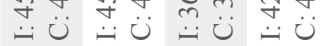

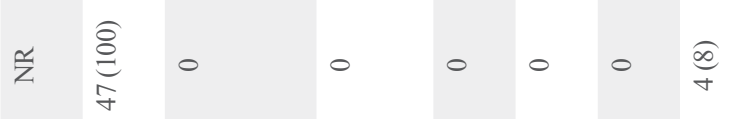

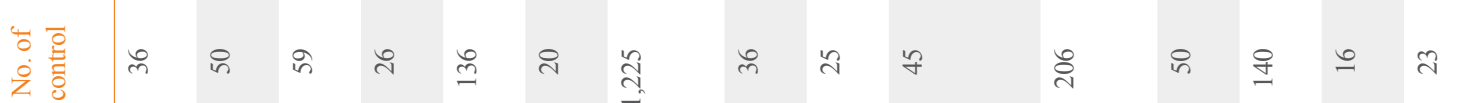
虫 A ก กิ กิ กิ

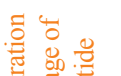



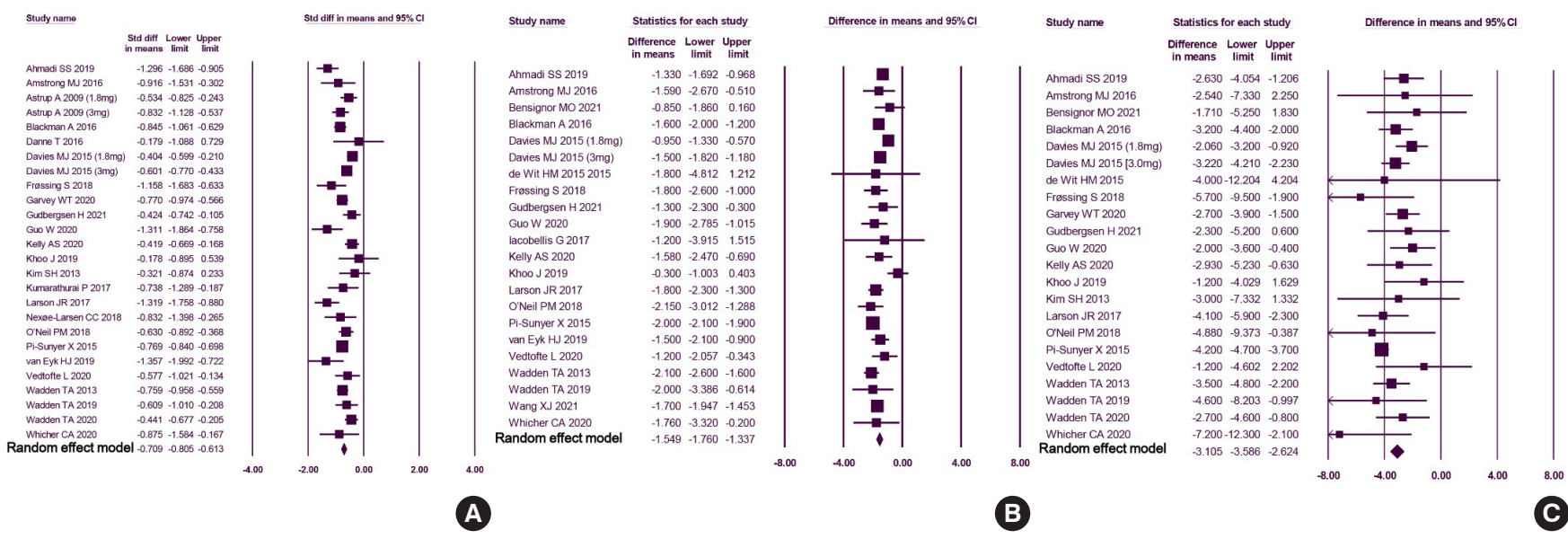

Fig. 2. Forest plots summarizing the effect of liraglutide on change of anthropometric data from baseline compared to placebo group. (A) Weight change (standard mean difference), (B) body mass index, $(\mathrm{C})$ waist circumference $(\mathrm{cm})$. CI, confidence interval.

in weight from the baseline and showed significantly decreased weight (MD, $-4.16 \% ; 95 \% \mathrm{CI},-4.90$ to $-3.43, I^{2}=72 \%$ ). In the subgroup analysis according to the dosage of liraglutide, the subgroup with seven studies of liraglutide administered at 1.8 $\mathrm{mg}$ /day showed a significant reduction in body weight (MD, $-4.04 \mathrm{~kg}$; $95 \% \mathrm{CI},-4.61$ to -3.47 ), and the subgroup with 12 studies with liraglutide administered at $3.0 \mathrm{mg} /$ day showed a significant change in body weight (MD, $-4.24 \mathrm{~kg}$; $95 \% \mathrm{CI}$, -5.27 to -3.22 ) (Table 2). Subgroup analysis was conducted according to diabetic status. In studies conducted among nondiabetic participants, patients receiving liraglutide had a significantly larger reduction in weight from the baseline (MD, -4.47 $\mathrm{kg} ; 95 \% \mathrm{CI},-5.38$ to -3.51$)$ than patients receiving placebo. Moreover, the patients with diabetes lost weight compared with the baseline weight (MD, $-3.78 \mathrm{~kg} ; 95 \% \mathrm{CI},-4.68$ to -3.23 ) (Table 3).

Twenty one studies with 6,870 participants (liraglutide group 4,230; placebo group 2,640) reported BMI changes from the baseline [12-14,16-18,20,21,23,25,27-29,32-39]. BMI was significantly reduced in the liraglutide group compared with the placebo group (MD, -1.55 ; 95\% CI, -1.76 to -1.34 ) (Fig. 2) with significant heterogeneity $\left(I^{2}=71.4 \%\right)$. Since analysis with the funnel plot was asymmetric (Egger's test $P=0.01$ ), the trimand fill method to adjust for publication bias was conducted. Three studies were imputed using the trim and fill method, but the significance was maintained (MD, $-1.47 ; 95 \% \mathrm{CI},-1.69$ to $-1.26)$ (Supplemental Fig. S1). In sensitivity analysis, the significance of the results did not change even after each study was removed, and no outliers were observed (Supplemental Fig. $\mathrm{S} 2$ ). The subgroups receiving either liraglutide 1.8 or $3.0 \mathrm{mg} /$ day showed a significant decrease in BMI (Table 2). The subgroup analysis according to the diabetes status showed that each subgroup had a significant reduction in BMI without heterogeneity (Table 3).

Twenty one studies with 7,437 participants (liraglutide group 4,509; placebo group 2,928) reported WC changes from the baseline [10-13,16-18,20,21,25,27-30,32-34,36-39]. The WC was significantly reduced in the liraglutide group compared with the placebo group (MD $-3.11 \mathrm{~cm} ; 95 \% \mathrm{CI},-3.59$ to -2.62 ) (Fig. 2) without significant heterogeneity among the studies $\left(I^{2}=34.3 \%\right)$. The funnel plot was symmetrical, and publication bias was not detected (Egger's test $P=0.14$ ) (Supplemental Fig. $\mathrm{S} 1)$. The MD was significant even when each study was removed from the sensitivity analysis, and no outliers were observed (Supplemental Fig. S2). In the subgroup analysis according to the dosage of liraglutide, the subgroup with 10 studies with the administration of liraglutide at $1.8 \mathrm{mg}$ /day showed a significant reduction in WC (MD, $-2.55 \mathrm{~cm}$; 95\% CI, -3.21 to -1.89 ), and the subgroup with 12 studies of liraglutide administration at $3.0 \mathrm{mg} /$ day also showed a significant change in WC (MD, $-3.39 \mathrm{~cm}$; 95\% CI, -3.93 to -2.85 ) (Table 2). In the subgroup analysis by diabetes status, patients receiving liraglutide had a significantly larger reduction in WC from baseline than patients receiving placebo in the subgroups without or with diabetes mellitus (DM) (patients without DM: MD, $-3.96 \mathrm{~cm}$; 95\% $\mathrm{CI},-4.37$ to -3.54 ; and patients with: $\mathrm{MD},-2.61 \mathrm{~cm} ; 95 \% \mathrm{CI}$, -3.14 to -2.07 ) (Table 3 ).

\section{Effect of liraglutide on cardiometabolic parameters}

Twenty-one studies with 7,529 participants (liraglutide group 

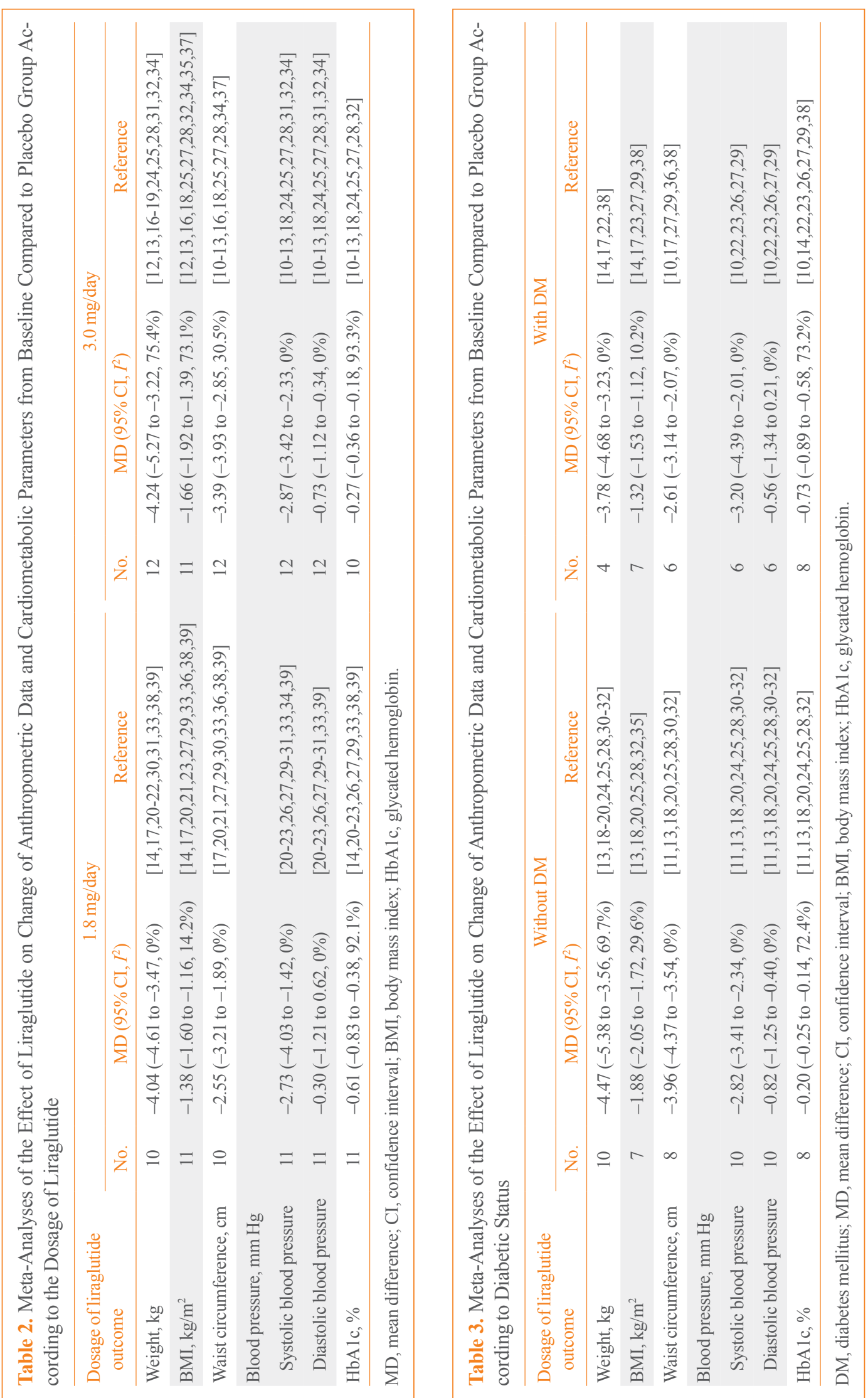

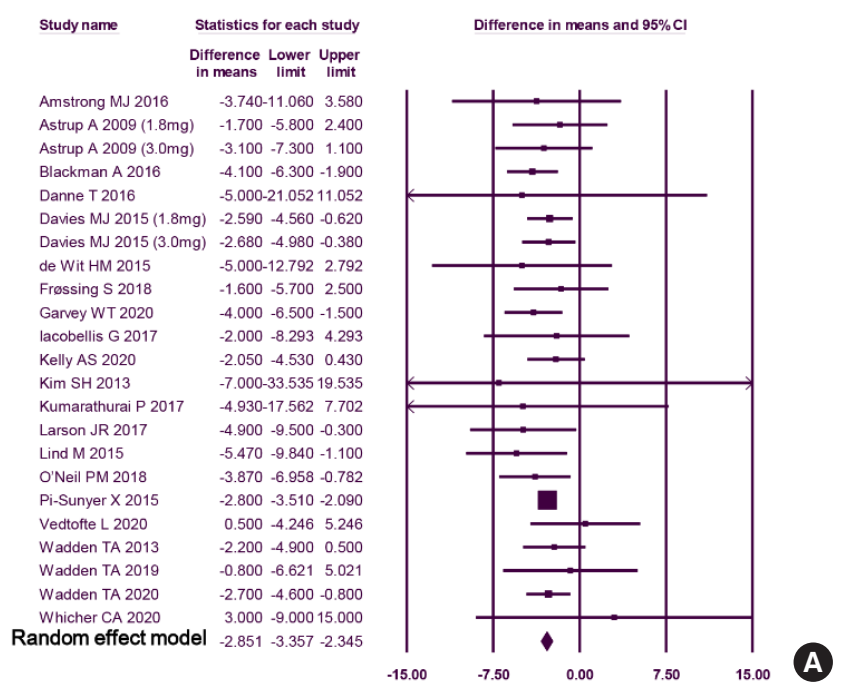

Study name
Amstrong MJ 2016
Blackman A 2016
Danne T 2016
Davies MJ 2015 (1.8mg)
Davies MJ 2015 (3.0mg)
de Wit HM 2015
Frossing S 2018
Garvey WT 2020
Guo W 2020
lacobellis G 2017
Kelly AS 2020
Kumarathurai P 2017
Larson JR 2017
Lind M 2015
O'Neil PM 2018
Pi-Sunyer X 2015
van Eyk HJ 2019 2019
Vedtofte L 2020
Wadden TA 2013
Wadden TA 2019
Wadden TA 2020
Random effect model

Difference in means and $95 \% \mathrm{Cl}$

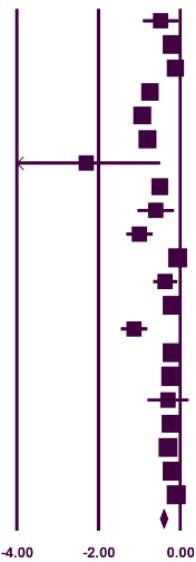

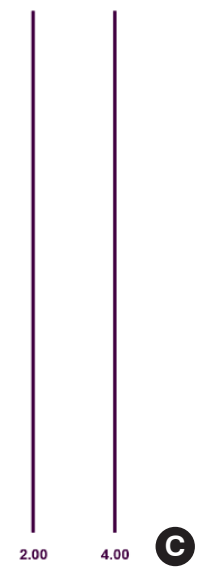

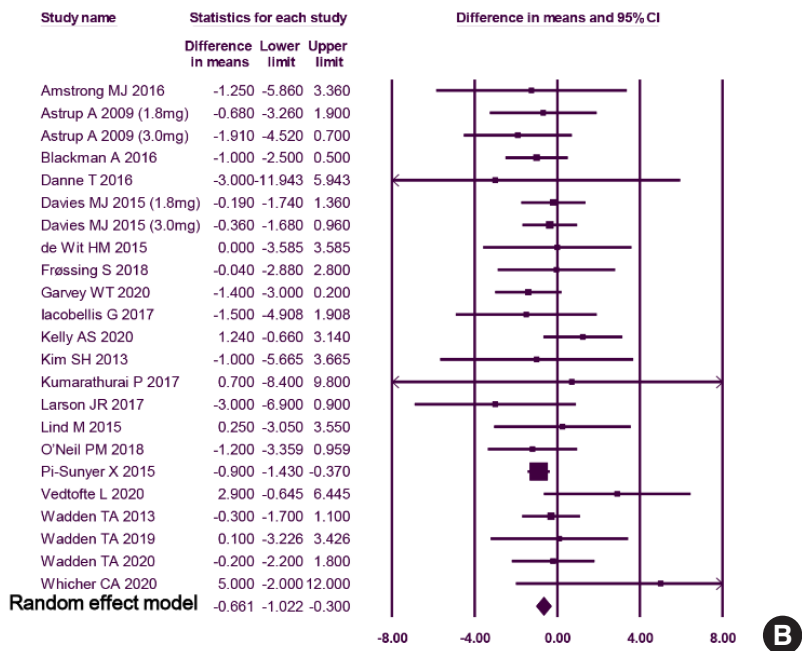

Study name Statistics for each study
Difference Lower Upper
in means limit limit -4.872-24.091 14.346 $-0.387-10.82710 .054$ $15.468-1.04331 .979$ $-3.867-22.08214 .348$ $-3.094-22.89616 .709$

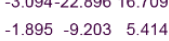
$-1.934-11.430 \quad 7.563$ $\begin{array}{lll}-6.574-17.015 & 3.867\end{array}$ $\begin{array}{lll}-3.867-14.910 & 7.176\end{array}$ $\begin{array}{lll}-3.867 & -14.910 & 7.176 \\ -3.480 & -7.734 & 0.773\end{array}$ $\begin{array}{lll}-6.300-14.893 & 2.293\end{array}$ $\begin{array}{lll}-2.707 & -7.734 & 2.320\end{array}$ $\begin{array}{lll}-2.905 & -5.284 & -0.526\end{array}$

Difference in means and $95 \% \mathrm{Cl}$

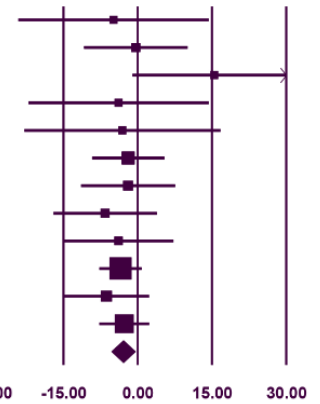

D

Fig. 3. Forest plots summarizing the effect of liraglutide on change of cardiometabolic parameters from baseline compared to placebo group. (A) Systolic blood pressure ( $\mathrm{mm} \mathrm{Hg}$ ), (B) diastolic blood pressure ( $\mathrm{mm} \mathrm{Hg}$ ), (C) glycated hemoglobin (\%), (D) low-density lipoprotein cholesterol (mg/dL). CI, confidence interval.

4,622; placebo group 2,907) reported changes in blood pressure from the baseline (Fig. 3) [10-13,17,20-34,39]. Liraglutide significantly lowered blood pressure without significant heterogeneity (systolic blood pressure: $\mathrm{MD},-2.85 \mathrm{~mm} \mathrm{Hg}$; 95\% CI, -3.36 to $-2.35 ; I^{2}=0 \%$; and diastolic blood pressure: MD, $-0.66 \mathrm{~mm} \mathrm{Hg} ; 95 \% \mathrm{CI},-1.02$ to $-0.30 ; I^{2}=0 \%$ ) (Fig. 3). The funnel plot was symmetrical, and no publication bias was detected (systolic blood pressure, Egger's test $P=0.91$; diastolic blood pressure, Egger's test $P=0.21$ ) (Supplemental Fig. S1). The significance of these results did not change even after each study was removed in the sensitivity analysis (Supplemental Fig. S2). In the subgroup analysis according to the dosage of liraglutide, the subgroup with 11 studies with a liraglutide dosage of $3.0 \mathrm{mg} /$ day showed a significant reduction in systolic blood pressure (MD, $-2.87 \mathrm{~mm} \mathrm{Hg}$; 95\% CI, -3.42 to -2.33 ) (Table 2 ), and the subgroup with nine studies with a liraglutide dosage of $1.8 \mathrm{mg} /$ day also showed a significant reduction in systolic blood pressure (MD, $-2.72 \mathrm{~mm} \mathrm{Hg}$; 95\% CI, -4.03 to -1.42 ). In the subgroup analysis according to the diabetes status, both subgroups among patients receiving liraglutide had a significant reduction of systolic blood pressure (Table 3). Diastolic blood pressure was significantly reduced only in the subgroup that received $3.0 \mathrm{mg}$ liraglutide/day or in the subgroup without DM (Tables 2, 3).

Twenty studies with 7,271 participants (liraglutide group 4,452; placebo group 2,819) reported changes in HbA1c (Fig. 3) [10-14,18,20-29,32,33,38,39]. Liraglutide significantly lowered $\mathrm{HbA1c}$, although there was significant heterogeneity (MD, 
Table 4. Adverse Events

\begin{tabular}{|c|c|c|c|c|c|}
\hline \multirow{2}{*}{ Adverse event } & \multicolumn{2}{|c|}{ Prevalence of adverse event } & \multirow{2}{*}{$\begin{array}{l}\text { Odds ratio } \\
(95 \% \mathrm{CI})\end{array}$} & \multirow{2}{*}{$\begin{array}{l}\text { Heterogeneity } \\
\qquad\left(I^{2}\right), \%\end{array}$} & \multirow{2}{*}{ Reference } \\
\hline & Liraglutide & Placebo & & & \\
\hline Serious adverse events & $6.1(279 / 4,587)$ & $5.6(155 / 2,759)$ & $1.10(0.89-1.36)$ & 0 & {$[10-13,18,19,21,23-28,31,33,34,36-38]$} \\
\hline $\begin{array}{l}\text { Treatment withdrawal } \\
\text { due to adverse events }\end{array}$ & $9.0(429 / 4,777)$ & $3.6(106 / 2,916)$ & $2.44(1.95-3.06)$ & 0 & {$[1,10-13,16,18-20,23-26,29-31,33,36,37,41]$} \\
\hline $\begin{array}{l}\text { Gastrointestinal adverse } \\
\text { events }\end{array}$ & $67.4(1,368 / 2,031)$ & $43.9(639 / 1,454)$ & $2.99(2.57-3.47)$ & 48.8 & {$[10-13,18,19,24-26,30,31,33,38,41]$} \\
\hline Nausea & $39.4(1,849 / 4,689)$ & $14.2(406 / 2,859)$ & $4.00(3.53-4.53)$ & 41.1 & {$[1,10-13,15,16,18,20,21,25,26,30-33,36,41]$} \\
\hline Diarrhea & $20.4(958 / 4,689)$ & $10.9(312 / 2,859)$ & $2.13(1.85-2.46)$ & 0 & {$[1,10-13,15,16,18,20,21,25,26,30-33,36,41]$} \\
\hline Vomiting & $16.1(741 / 4,610)$ & $4.6(127 / 2,785)$ & $4.03(3.30-4.93)$ & 12.4 & {$[1,10-13,15,18,20,21,25,30-33,36,41]$} \\
\hline Abdominal pain & $6.8(277 / 4,071)$ & $4.7(111 / 2,387)$ & $1.62(1.28-2.06)$ & 43.9 & {$[1,10-13,15,16,20,21,30,32,33,36,41]$} \\
\hline Hypoglycemia & $36.7(478 / 1,303)$ & $26.0(237 / 910)$ & $1.66(1.32-2.09)$ & 38.6 & {$[10,11,18,21,24,32,36]$} \\
\hline
\end{tabular}

Values are expressed as percentage (number/total number).

CI, confidence interval.

$-0.40 \%$; $95 \%$ CI, -0.49 to $-0.31 ; I^{2}=93.2 \%$ ) (Fig. 3 ). The funnel plot was asymmetrical, and significant publication bias was found (Egger's test $P=0.03$ ) (Supplemental Fig. S1). One study was imputed using the trim and fill method, but the significance was maintained (MD, $-0.40 \% ; 95 \% \mathrm{CI},-0.49$ to -0.31 ). The significance of the results did not change following removal of each study in the sensitivity analysis, and no outliers were observed (Supplemental Fig. S2). In the subgroup analysis according to the dosage of liraglutide, the subgroup with 10 studies with a liraglutide dosage of $3.0 \mathrm{mg} /$ day showed a significant reduction in HbA1c (MD, $-0.27 \%$; 95\% CI, -0.36 to -0.18 ) (Table 2), and the subgroup with 11 studies of liraglutide dosage at $1.8 \mathrm{mg}$ /day also showed a significant reduction in $\mathrm{HbA} 1 \mathrm{c}$ (MD, $-0.61 \%$; $95 \% \mathrm{CI},-0.83$ to -0.38$)$. Among patients with diabetes, the HbA1c decreased by $0.73 \%(95 \% \mathrm{CI},-0.89$ to -0.58$)$ compared with the placebo group. Furthermore, improvements in $\mathrm{HbAlc}$ were greater in studies in patients with diabetes than in studies among patients without diabetes (MD, $-0.20 \%$; 95\% CI, -0.25 to -0.14 ) (Table 3 ).

Twelve studies with 1,369 participants (liraglutide group 693; placebo group 676) reported changes in LDL-C concentration $(\mathrm{mg} / \mathrm{dL})$ from the baseline [11,13-15,20,22,26,30,32,33,38,39]. The MD between the liraglutide group and the placebo group using a random effects model was $-2.91 \mathrm{mg} / \mathrm{dL}(95 \% \mathrm{CI},-5.28$ to -0.53$)$ without significant heterogeneity $\left(I^{2}=0 \%\right)$. The funnel plot was symmetrical, and publication bias was not detected (Egger's test $P=0.44$ ) (Supplemental Fig. S1). In sensitivity analysis, the significance of the results did not change even after each study was removed, and no outliers were observed (Sup- plemental Fig. S2). Six studies with 5,481 participants (liraglutide group 3,535; placebo group 1,946) reported percentage changes in LDL-C concentration ( $\%$ ) from baseline and showed a significant reduction in LDL-C concentration (MD, $-0.87 \%$; $95 \% \mathrm{CI},-1.17$ to -0.56$)$ [27].

\section{Safety of liraglutide}

Among 4,587 participants with liraglutide in 19 studies [1013,18,19,21,23-28,31,33,34,36-38], 279 (6.1\%) had serious adverse event (Table 4). However, the risk in the liraglutide group for serious adverse event was not significantly higher than that of the placebo group (odds ratio [OR], 1.10; 95\% CI, 0.89 to 1.36; $\left.I^{2}=0 \%\right)$. In 20 studies with 4,777 participants, $429(9.0 \%)$ discontinued the treatment due to adverse events $[1,10-13,16$, 18-20,23-26,29-31,33,36,37,41].

In 14 studies with 3,485 participants (liraglutide group 2,031; placebo group 1,454) [10-13,18,19,24-26,30,31,33,38,41], participants who received liraglutide had a significantly higher risk of gastrointestinal symptoms than those in the placebo group (OR, 2.99; 95\% CI, 2.57 to $3.47 ; I^{2}=48.8 \%$ ). The risk of the liraglutide group for hypoglycemia was significantly higher than that of the placebo group (OR, 1.66; 95\% CI, 1.32 to 2.09; $\left.I^{2}=38.6 \%\right)[10,11,18,21,24,32,36]$.

\section{DISCUSSION}

In this meta-analysis of 31 studies, liraglutide therapy showed a significant association with body weight change. Overweight and obese people treated with liraglutide showed reduced body 
weight and decreased WC compared with those treated with placebo. Moreover, liraglutide reduced both systolic and diastolic blood pressure, improved glucose tolerance, and improved dyslipidemia.

Since the FDA approved liraglutide as a long-term anti-obesity drug in 2014, researchers have analyzed the clinical outcomes of liraglutide and compared its benefits to other anti-obesity drugs. In 2016, Khera et al. [5] compared the weight loss efficacy of five FDA approved drugs in 28 clinical trials by metaanalysis. The primary outcome of the study was the proportion of participants who achieved at least 5\% weight loss at 1 year [5]. They reported that liraglutide was one of the two most effective weight loss drugs (liraglutide: average $5.3 \mathrm{~kg}$ weight loss at 1 year compared with placebo) [5]. The most effective drug was phentermine-topiramate (average $8.8 \mathrm{~kg}$ loss at 1 year). Both of these drugs are appetite suppressants. A recent longterm follow-up study reported that liraglutide also led to sustained weight loss. A 3-year clinical trial reported that participants receiving liraglutide maintained reduced body weight at 3 years [34]. After 5 years, patients with type 2 diabetes mellitus (T2DM) receiving liraglutide showed significantly reduced body weight $(-5.3 \pm 6.4 \mathrm{~kg})$ [42].

Liraglutide has also shown significant improvements in cardiovascular outcomes in T2DM patients. The Liraglutide and Cardiovascular Outcomes in Type 2 Diabetes (LEADER) trial (Clinicaltrials.gov NCT01179048) reported that liraglutide reduced major cardiovascular outcomes (hazard ratio, 0.85; 95\% CI, 0.73 to 0.99 ) in T2DM patients with myocardial infarction/ stroke history [43]. Furthermore, liraglutide reduced the composite risk of heart failure (HF) hospitalization or cardiovascular death in T2DM patients with and without HF (hazard ratio, $0.92 ; 95 \% \mathrm{CI}, 0.74$ to 1.15 ) and those without a history of HF (hazard ratio, $0.77 ; 95 \% \mathrm{CI}, 0.65$ to 0.91 ) [44].

In patients without diabetes, liraglutide does not reduce the cardiovascular disease risk. The Satiety and Clinical AdiposityLiraglutide Evidence (SCALE) clinical trial reported that 3.0 mg liraglutide had no significant association with the composite outcome of first occurrence of cardiovascular death, nonfatal myocardial infraction, or nonfatal stroke (hazard ratio, 0.70; 95\% CI, 0.20 to 2.50) [41]. Although that study did not prove the benefits of liraglutide on major cardiovascular outcomes, this meta-analysis showed the possible cardiovascular benefits of liraglutide in nondiabetic individuals. Liraglutide treatment significantly reduced both systolic and diastolic blood pressures and LDL-C in nondiabetic participants.

The underlying mechanism of body weight reduction by lira- glutide mainly depends on appetite suppression and delayed gastric emptying by GLP-1 [7]. The half-life of natural GLP-1 in the circulation is less than 2 minutes [7]. The enzyme dipeptidyl peptidase-4 degrades GLP-1, and the kidneys rapidly clear the remnant metabolites [45]. To overcome this limitation, liraglutide was created by substituting an amino acid and adding a fatty acid chain [45]. The half-life of liraglutide is 13 hours, which means that once-daily subcutaneous administration is sufficient to control the glucose levels of people with diabetes and to reduce the appetite of obese individuals [45].

GLP-1 receptor agonists have shown non-inferiority for cardiovascular outcomes of T2DM patients in seven recent clinical trials; Evaluation of Lixisenatide in Acute Coronary Syndrome (ELIXA); LEADER; Semaglutide Unabated Sustainability in Treatment of Type 2 Diabetes (SUSTAIN-6); Exenatide Study of Cardiovascular Event Lowering Trial (EXSCEL); Harmony outcomes study; Researching Cardiovascular Events With a Weekly Incretin in Diabetes (REWIND); and Peptide Innovation for Early Diabetes Treatment (PIONEER) 6 trials [46]. Among these clinical trials, GLP-1 receptor agonists showed significant cardiovascular benefits in two trials (liraglutide in the LEADER trial and semaglutide in SUSTAIN-6) [46]. Liraglutide improved cardiovascular outcomes in T2DM patients (hazard ratio, 0.87 ; 95\% CI, 0.78 to 0.97 ) over a 3.8-year median follow-up [46,47]. Semaglutide is a new, long-acting GLP-1 receptor agonist, which allows for weekly subcutaneous injection [48]. Moreover, semaglutide improved cardiovascular outcomes in T2DM patients (hazard ratio, 0.74; 95\% CI, 0.58 to 0.95 ) during a 2.1-year median follow-up [46,49].

This meta-analysis provides supporting evidence of the cardiovascular benefits of GLP-1 receptor agonists. The underlying cardioprotective mechanisms of liraglutide depend on both direct and indirect actions in multiple organs. GLP-1 receptor agonists have direct effects on the heart, as well as broader antidiabetic and anti-obesity effects [50]. GLP-1 reduces cardiac inflammation in mice receiving a high-fat diet and reduced the infarct size in a mouse myocardial infarction model [51]. In animal studies and human clinical trials, GLP-1 receptor agonists have resulted in improved lipid profiles, reduce fat inflammation, and induce natriuresis in the kidney [51,52].

This meta-analysis show that liraglutide treatment reduced the LDL-C levels of the participants. This improvement may be the result of body weight reduction and improved glucose control by liraglutide treatment [52]. Recent papers reported that GLP-1 receptor agonism directly modulates hepatic cholesterol metabolism by suppressing adenosine triphosphate-binding cas- 
sette transporter A1 [53] and regulating intestinal lipid and lipoprotein metabolism [54]. These direct effects of GLP-1 are other possible mechanisms of lipid lowering effects of liraglutide.

This study had some limitations. First, we could not evaluate the direct benefits of liraglutide use on the major cardiovascular outcomes of participants. Although, we identified enough data related to cardiovascular parameters and calculated the beneficial effects of liraglutide use on these parameters, there are enough clinical studies about cardiovascular events after liraglutide use. Second, we could not analyze important blood parameters related to obesity and cardiometabolic dysfunctions, such as adiponectin, leptin, and inflammatory markers, because of lack of data. Further long-term follow-up clinical trials are needed to overcome these limitations.

In conclusion, liraglutide is a very effective treatment for overweight and obese individuals for body weight reduction and sustained weight loss. Furthermore, this therapy improves cardiometabolic parameters during treatment. Additional longterm clinical studies are needed to confirm the cardioprotective role of liraglutide in nondiabetic individuals.

\section{CONFLICTS OF INTEREST}

No potential conflict of interest relevant to this article was reported.

\section{ACKNOWLEDGMENTS}

This research was supported by the Basic Science Research Program through the National Research Foundation of Korea (NRF) funded by the Ministry of Education (2016R1A6A3A04010466, 2020R1C1C1004999 to Chang-Myung Oh).

\section{AUTHOR CONTRIBUTIONS}

Conception or design: S.M., S.H.Y., C.M.O. Acquisition, analysis, or interpretation of data: S.M., J.L., Y.J.K., C.M.O. Drafting the work or revising: S.M., H.S.C., S.H.Y., C.M.O. Final approval of the manuscript: S.M., H.S.C., J.M.Y., S.H.Y., C.M.O.

\section{ORCID}

Shinje Moon https://orcid.org/0000-0003-3298-3630

Sung Hoon Yu https://orcid.org/0000-0003-4391-4526

Chang-Myung Oh https://orcid.org/0000-0001-6681-4478

\section{REFERENCES}

1. Pi-Sunyer X. The medical risks of obesity. Postgrad Med 2009;121:21-33.

2. GBD 2015 Obesity Collaborators, Afshin A, Forouzanfar MH, Reitsma MB, Sur P, Estep K, et al. Health effects of overweight and obesity in 195 countries over 25 years. N Engl J Med 2017;377:13-27.

3. Wilson K. Obesity: lifestyle modification and behavior interventions. FP Essent 2020;492:19-24.

4. Dalle Grave R, Calugi S, Centis E, Marzocchi R, El Ghoch $\mathrm{M}$, Marchesini G. Lifestyle modification in the management of the metabolic syndrome: achievements and challenges. Diabetes Metab Syndr Obes 2010;3:373-85.

5. Khera R, Murad MH, Chandar AK, Dulai PS, Wang Z, Prokop LJ, et al. Association of pharmacological treatments for obesity with weight loss and adverse events: a systematic review and meta-analysis. JAMA 2016;315:2424-34.

6. Bode B. Liraglutide: a review of the first once-daily GLP-1 receptor agonist. Am J Manag Care 2011;17(2 Suppl):S59-70.

7. Mehta A, Marso SP, Neeland IJ. Liraglutide for weight management: a critical review of the evidence. Obes Sci Pract 2017;3:3-14.

8. Howell R, Wright AM, Clements JN. Clinical potential of liraglutide in cardiovascular risk reduction in patients with type 2 diabetes: evidence to date. Diabetes Metab Syndr Obes 2019;12:505-12.

9. Moher D, Liberati A, Tetzlaff J, Altman DG; PRISMA Group. Preferred reporting items for systematic reviews and meta-analyses: the PRISMA statement. PLoS Med 2009;6: e1000097.

10. Garvey WT, Birkenfeld AL, Dicker D, Mingrone G, Pedersen SD, Satylganova A, et al. Efficacy and safety of liraglutide $3.0 \mathrm{mg}$ in individuals with overweight or obesity and type 2 diabetes treated with basal insulin: the SCALE insulin randomized controlled trial. Diabetes Care 2020;43:1085-93.

11. Wadden TA, Tronieri JS, Sugimoto D, Lund MT, Auerbach P, Jensen C, et al. Liraglutide $3.0 \mathrm{mg}$ and intensive behavioral therapy (ibt) for obesity in primary care: the SCALE IBT randomized controlled trial. Obesity (Silver Spring) 2020;28:529-36.

12. Kelly AS, Auerbach P, Barrientos-Perez M, Gies I, Hale $\mathrm{PM}$, Marcus $\mathrm{C}$, et al. A randomized, controlled trial of liraglutide for adolescents with obesity. N Engl J Med 2020; 382:2117-28.

13. Wadden TA, Walsh OA, Berkowitz RI, Chao AM, Alamud- 
din N, Gruber K, et al. Intensive behavioral therapy for obesity combined with liraglutide $3.0 \mathrm{mg}$ : a randomized controlled trial. Obesity (Silver Spring) 2019;27:75-86.

14, van Eyk HJ, Blauw LL, Bizino MB, Wang Y, van Dijk KW, de Mutsert R, et al. Hepatic triglyceride content does not affect circulating CETP: lessons from a liraglutide intervention trial and a population-based cohort. Sci Rep 2019;9: 9996.

15. Peradze N, Farr OM, Perakakis N, Lazaro I, Sala-Vila A, Mantzoros CS. Short-term treatment with high dose liraglutide improves lipid and lipoprotein profile and changes hormonal mediators of lipid metabolism in obese patients with no overt type 2 diabetes mellitus: a randomized, placebocontrolled, cross-over, double-blind clinical trial. Cardiovasc Diabetol 2019;18:141.

16. Khoo J, Hsiang JC, Taneja R, Koo SH, Soon GH, Kam CJ, et al. Randomized trial comparing effects of weight loss by liraglutide with lifestyle modification in non-alcoholic fatty liver disease. Liver Int 2019;39:941-9.

17. Ahmadi SS, Filipsson K, Dimenas H, Isaksson SS, Imberg $\mathrm{H}$, Sjoberg S, et al. Effect of liraglutide on anthropometric measurements, sagittal abdominal diameter and adiponectin levels in people with type 2 diabetes treated with multiple daily insulin injections: evaluations from a randomized trial (MDI-liraglutide study 5). Obes Sci Pract 2019;5:130-40.

18. O’Neil PM, Birkenfeld AL, McGowan B, Mosenzon O, Pedersen SD, Wharton S, et al. Efficacy and safety of semaglutide compared with liraglutide and placebo for weight loss in patients with obesity: a randomised, double-blind, placebo and active controlled, dose-ranging, phase 2 trial. Lancet 2018;392:637-49.

19. Nexoe-Larsen CC, Sorensen PH, Hausner H, Agersnap M, Baekdal M, Bronden A, et al. Effects of liraglutide on gallbladder emptying: a randomized, placebo-controlled trial in adults with overweight or obesity. Diabetes Obes Metab 2018;20:2557-64.

20. Frossing S, Nylander M, Kistorp C, Skouby SO, Faber J. Effect of liraglutide on atrial natriuretic peptide, adrenomedullin, and copeptin in PCOS. Endocr Connect 2018;7:115-23.

21. Larsen JR, Vedtofte L, Jakobsen MS, Jespersen HR, Jakobsen MI, Svensson CK, et al. Effect of liraglutide treatment on prediabetes and overweight or obesity in clozapine- or olanzapine-treated patients with schizophrenia spectrum disorder: a randomized clinical trial. JAMA Psychiatry 2017;74:719-28.

22. Kumarathurai P, Anholm C, Larsen BS, Olsen RH, Madsbad
$\mathrm{S}$, Kristiansen $\mathrm{O}$, et al. Effects of liraglutide on heart rate and heart rate variability: a randomized, double-blind, placebo-controlled crossover study. Diabetes Care 2017;40: 117-24.

23. Iacobellis G, Mohseni M, Bianco SD, Banga PK. Liraglutide causes large and rapid epicardial fat reduction. Obesity (Silver Spring) 2017;25:311-6.

24. Danne T, Biester T, Kapitzke K, Jacobsen SH, Jacobsen LV, Petri KC, et al. Liraglutide in an adolescent population with obesity: a randomized, double-blind, placebo-controlled 5-week trial to assess safety, tolerability, and pharmacokinetics of liraglutide in adolescents aged 12-17 years. J Pediatr 2017;181:146-53.

25. Blackman A, Foster GD, Zammit G, Rosenberg R, Aronne L, Wadden T, et al. Effect of liraglutide $3.0 \mathrm{mg}$ in individuals with obesity and moderate or severe obstructive sleep apnea: the SCALE Sleep Apnea randomized clinical trial. Int J Obes (Lond) 2016;40:1310-9.

26. Lind M, Hirsch IB, Tuomilehto J, Dahlqvist S, Ahren B, Torffvit $\mathrm{O}$, et al. Liraglutide in people treated for type 2 diabetes with multiple daily insulin injections: randomised clinical trial (MDI Liraglutide trial). BMJ 2015;351:h5364.

27. Davies MJ, Bergenstal R, Bode B, Kushner RF, Lewin A, Skjoth TV, et al. Efficacy of liraglutide for weight loss among patients with type 2 diabetes: the SCALE diabetes randomized clinical trial. JAMA 2015;314:687-99.

28. Pi-Sunyer X, Astrup A, Fujioka K, Greenway F, Halpern A, Krempf M, et al. A randomized, controlled trial of $3.0 \mathrm{mg}$ of liraglutide in weight management. N Engl J Med 2015;373: 11-22.

29. de Wit HM, Vervoort GM, Jansen HJ, de Galan BE, Tack CJ. Durable efficacy of liraglutide in patients with type 2 diabetes and pronounced insulin-associated weight gain: 52week results from the Effect of Liraglutide on insulin-associated wEight GAiN in patients with Type 2 diabetes' (ELEGANT) randomized controlled trial. J Intern Med 2016;279: 283-92.

30. Kim SH, Abbasi F, Lamendola C, Liu A, Ariel D, Schaaf P, et al. Benefits of liraglutide treatment in overweight and obese older individuals with prediabetes. Diabetes Care 2013;36:3276-82.

31. Astrup A, Rossner S, Van Gaal L, Rissanen A, Niskanen L, Al Hakim M, et al. Effects of liraglutide in the treatment of obesity: a randomised, double-blind, placebo-controlled study. Lancet 2009;374:1606-16.

32. Wadden TA, Hollander P, Klein S, Niswender K, Woo V, 
Hale PM, et al. Weight maintenance and additional weight loss with liraglutide after low-calorie-diet-induced weight loss: the SCALE Maintenance randomized study. Int J Obes (Lond) 2013;37:1443-51.

33. Armstrong MJ, Gaunt P, Aithal GP, Barton D, Hull D, Parker R, et al. Liraglutide safety and efficacy in patients with non-alcoholic steatohepatitis (LEAN): a multicentre, double-blind, randomised, placebo-controlled phase 2 study. Lancet 2016;387:679-90.

34. Whicher CA, Price HC, Phiri P, Rathod S, Barnard-Kelly K, Ngianga $\mathrm{K}$, et al. The use of liraglutide $3.0 \mathrm{mg}$ daily in the management of overweight and obesity in people with schizophrenia, schizoaffective disorder and first episode psychosis: results of a pilot randomized, double-blind, placebocontrolled trial. Diabetes Obes Metab 2021;23:1262-71.

35. Wang XJ, Gong P, Zhou C, Huang C, Lok UW, Tang S, et al. Liraglutide reduces attenuation coefficient as a measure of hepatic steatosis during 16 weeks' treatment in nondiabetic obese patients: a pilot trial. JGH Open 2020;5:193-8.

36. Bensignor MO, Bomberg EM, Bramante CT, Divyalasya TV, Hale PM, Ramesh CK, et al. Effect of liraglutide treatment on body mass index and weight parameters in children and adolescents with type 2 diabetes: post hoc analysis of the ellipse trial. Pediatr Obes 2021 Feb 25 [Epub]. https:// doi.org/10.1111/ijpo.12778.

37. Gudbergsen H, Overgaard A, Henriksen M, Waehrens EE, Bliddal H, Christensen R, et al. Liraglutide after diet-induced weight loss for pain and weight control in knee osteoarthritis: a randomized controlled trial. Am J Clin Nutr 2021; 113:314-23.

38. Guo W, Tian W, Lin L, Xu X. Liraglutide or insulin glargine treatments improves hepatic fat in obese patients with type 2 diabetes and nonalcoholic fatty liver disease in twenty-six weeks: a randomized placebo-controlled trial. Diabetes Res Clin Pract 2020;170:108487.

39. Vedtofte L, Bahne E, Foghsgaard S, Bagger JI, Andreasen C, Strandberg C, et al. One year's treatment with the glucagonlike peptide 1 receptor agonist liraglutide decreases hepatic fat content in women with nonalcoholic fatty liver disease and prior gestational diabetes mellitus in a randomized, placebo-controlled trial. J Clin Med 2020;9:3213.

40. Tronieri JS, Wadden TA, Walsh O, Berkowitz RI, Alamuddin N, Gruber K, et al. Effects of liraglutide on appetite, food preoccupation, and food liking: results of a randomized controlled trial. Int J Obes (Lond) 2020;44:353-61.

41. Davies MJ, Aronne LJ, Caterson ID, Thomsen AB, Jacob- sen PB, Marso SP, et al. Liraglutide and cardiovascular outcomes in adults with overweight or obesity: a post hoc analysis from SCALE randomized controlled trials. Diabetes Obes Metab 2018;20:734-9.

42. Frison V, Simioni N, Marangoni A, Balzano S, Vinci C, Zenari L, et al. Clinical impact of 5 years of liraglutide treatment on cardiovascular risk factors in patients with type 2 diabetes mellitus in a real-life setting in Italy: an observational study. Diabetes Ther 2018;9:2201-8.

43. Verma S, Poulter NR, Bhatt DL, Bain SC, Buse JB, Leiter LA, et al. Effects of liraglutide on cardiovascular outcomes in patients with type 2 diabetes mellitus with or without history of myocardial infarction or stroke. Circulation 2018; 138:2884-94.

44. Marso SP, Baeres FM, Bain SC, Goldman B, Husain M, Nauck MA, et al. Effects of liraglutide on cardiovascular outcomes in patients with diabetes with or without heart failure. J Am Coll Cardiol 2020;75:1128-41.

45. Jackson SH, Martin TS, Jones JD, Seal D, Emanuel F. Liraglutide (victoza): the first once-daily incretin mimetic injection for type-2 diabetes. P\&T 2010;35:498-529.

46. Sheahan KH, Wahlberg EA, Gilbert MP. An overview of GLP-1 agonists and recent cardiovascular outcomes trials. Postgrad Med J 2020;96:156-61.

47. Marso SP, Daniels GH, Brown-Frandsen K, Kristensen P, Mann JF, Nauck MA, et al. Liraglutide and cardiovascular outcomes in type 2 diabetes. N Engl J Med 2016;375:31122.

48. Carlsson Petri KC, Ingwersen SH, Flint A, Zacho J, Overgaard RV. Semaglutide s.c. once-weekly in type 2 diabetes: a population pharmacokinetic analysis. Diabetes Ther 2018; 9:1533-47.

49. Marso SP, Bain SC, Consoli A, Eliaschewitz FG, Jodar E, Leiter LA, et al. Semaglutide and cardiovascular outcomes in patients with type 2 diabetes. N Engl J Med 2016;375:183444.

50. Lingvay I, Leiter LA. Use of GLP-1 RAs in cardiovascular disease prevention: a practical guide. Circulation 2018;137: 2200-2.

51. Drucker DJ. The cardiovascular biology of glucagon-like peptide-1. Cell Metab 2016;24:15-30.

52. Hasegawa Y, Hori M, Nakagami T, Harada-Shiba M, Uchigata Y. Glucagon-like peptide-1 receptor agonists reduced the low-density lipoprotein cholesterol in Japanese patients with type 2 diabetes mellitus treated with statins. J Clin Lipidol 2018;12:62-9. 
53. Yao Y, Li Q, Wang W, Zhang J, Gao P, Xu Y. Glucagon-like peptide-1 modulates cholesterol homeostasis by suppressing the miR-19b-induced downregulation of ABCA1. Cell Physiol Biochem 2018;50:679-93.
54. Mulvihill EE. Regulation of intestinal lipid and lipoprotein metabolism by the proglucagon-derived peptides glucagon like peptide 1 and glucagon like peptide 2. Curr Opin Lipidol 2018;29:95-103. 